Mathematical Research Letters 9, 633-638 (2002)

\title{
EVERY MORPHISM IS THE RESTRICTION OF A TORIC ONE
}

\author{
Florian Berchtold and JÜrgen Hausen
}

\begin{abstract}
We show that every morphism of divisorial prevarieties is the restriction of a toric morphism. This extends an embedding theorem of Włodarczyk to the setting of morphisms.
\end{abstract}

\section{Introduction}

A prevariety $X$ over an algebraically closed field $\mathbb{K}$ is called divisorial if every $x \in X$ has an affine open neighbourhood $X \backslash Z(f)$ obtained by removing the zero set $Z(f)$ of a global section $f$ of some line bundle on $X$, compare [2]. Nonseparated divisorial prevarieties occur for example in quotient constructions, where they behave more natural than their separated counterparts, see [4].

For divisorial prevarieties, Włodarczyk's Embedding Theorem [6, Theorem C] can be improved as follows: Every divisorial prevariety admits a closed embedding into a smooth toric prevariety with affine diagonal morphism, see [5, Theorem 3.2 and Remark 3.3]. In the present note, we extend this statement to morphisms.

The "ambient morphisms" will be toric morphisms $\psi: Z \rightarrow Z^{\prime}$, i.e., morphisms of toric prevarieties $Z$ and $Z^{\prime}$ that restrict to a homomorphism $T \rightarrow T^{\prime}$ of the respective big tori $T \subset Z$ and $T^{\prime} \subset Z^{\prime}$ and satisfy $\psi(t \cdot z)=\psi(t) \cdot \psi(z)$. The description of toric morphisms by combinatorial data is a powerful tool for explicit studies, see [3] and [1].

For the sake of a rounded picture, we formulate our result in terms of a refined concept of divisoriality: Given $k>0$, we say that a not necessarily irreducible prevariety $X$ is $k$-divisorial if for any collection $x_{1}, \ldots, x_{k} \in X$ there is a line bundle $L$ on $X$ and a global section $f: X \rightarrow L$ such that $X \backslash Z(f)$ is an affine neighbourhood of the points $x_{1}, \ldots, x_{k}$, compare also [5]. We prove:

Theorem. Let $\varphi: X \rightarrow Y$ be a morphism of $k$-divisorial prevarieties. Then there exist $k$-divisorial smooth toric prevarieties $Z_{X}, Z_{Y}$ and a commutative

Received August 20, 2001.

2000 Mathematics Subject Classification. 14E25, 14M25, 14C20. 
diagram

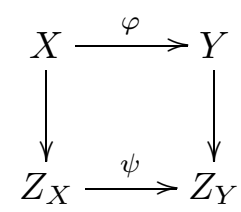

where the downwards arrows are closed embeddings and $\psi: Z_{X} \rightarrow Z_{Y}$ is a toric morphism.

Note that the theorem applies in particular to all $\mathbb{Q}$-factorial prevarieties with affine diagonal morphism. For $k>1$, all prevarieties occuring in the statement are in fact separated, and in this case the theorem can also be derived from the results of [5, Section 5] using the graph of the morphism $\varphi: X \rightarrow Y$.

\section{Proof of the Theorem}

Throughout the proof of the main result we shall make use of the methods developed in [5, Section 2]. The first step is to reduce the study of arbitrary morphisms to the study of morphisms of affine varieties. As this might be of independent interest, we give a separate statement:

Lemma. Let $X, Y$ be $k$-divisorial prevarieties, and let $\varphi: X \rightarrow Y$ be any morphism. Then there exist:

(i) algebraic tori $H_{X}, H_{Y}$, an affine $H_{X}$-variety $\bar{X}$ and an affine $H_{Y}$-variety $\bar{Y}$

(ii) open dense invariant subsets $\widehat{X} \subset \bar{X}$ and $\widehat{Y} \subset \bar{Y}$ where the respective tori act freely with geometric quotients

$$
q_{X}: \widehat{X} \rightarrow X, \quad q_{Y}: \widehat{Y} \rightarrow Y,
$$

(iii) a homomorphism $\widetilde{\varphi}: H_{X} \rightarrow H_{Y}$ of algebraic tori and a commutative diagram of morphisms

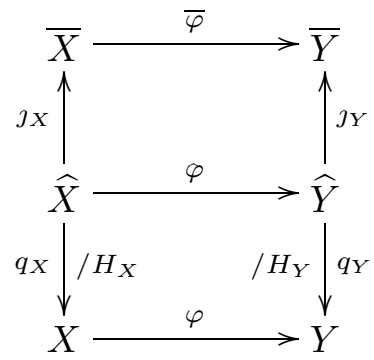

where $\bar{\varphi}$ is equivariant in the sense that $\bar{\varphi}(t \cdot x)=\widetilde{\varphi}(t) \cdot \bar{\varphi}(x)$ and the maps $\jmath_{X}, \jmath_{Y}$ denote the open inclusions,

(iv) regular $H_{Y}$-homogeneous functions $g_{1}, \ldots, g_{s} \in \mathcal{O}(\bar{Y})$ such that

(a) $\bar{Y}_{g_{j}}=q_{Y}^{-1}\left(Y \backslash Z\left(g_{j}\right)\right)$ holds for every $j=1, \ldots, s$,

(b) any collection $y_{1}, \ldots, y_{k} \in \widehat{Y}$ is contained in some $\bar{Y}_{g_{j}}$,

(v) regular $H_{X}$-homogeneous functions $f_{1}, \ldots, f_{r} \in \mathcal{O}(\bar{X})$ such that 
(a) $\bar{X}_{f_{i}}=q_{X}^{-1}\left(X \backslash Z\left(f_{i}\right)\right)$ holds for every $i=1, \ldots, r$,

(b) any collection $x_{1}, \ldots, x_{k} \in \widehat{X}$ is contained in some $\bar{X}_{f_{i}}$.

Proof. Choose line bundles $E_{1}, \ldots, E_{d}$ on $Y$ and sections $g_{j}: Y \rightarrow E_{j}$ such that the sets $Y_{j}:=Y \backslash Z\left(g_{j}\right)$ are affine and any collection $y_{1}, \ldots, y_{k}$ is contained in some $Y_{j}$. In doing this, we may assume that the line bundles generate a free abelian group $\Gamma$ in the sense of [5, Section 2]. For each $E \in \Gamma$, let $\mathcal{B}_{E}$ denote its sheaf of sections. Consider the associated $\Gamma$-graded $\mathcal{O}_{Y}$-algebra

$$
\mathcal{B}:=\bigoplus_{E \in \Gamma} \mathcal{B}_{E}
$$

This algebra gives rise to a prevariety $\widehat{Y}:=\operatorname{Spec}(\mathcal{B})$ and a canonical morphism $q_{Y}: \widehat{Y} \rightarrow Y$. The morphism $q_{Y}$ is a geometric quotient for the action of the algebraic torus $H_{Y}:=\operatorname{Spec}(\mathbb{K}[\Gamma])$ on $\widehat{Y}$ defined by the grading of $\mathcal{B}$. Note that $\widehat{Y}$ is quasiaffine; in fact, by [5, Lemma 2.4], there is an $H_{Y}$-equivariant affine closure $\bar{Y}$ of $\widehat{Y}$ such that the $g_{j}$ extend regularly to $\bar{Y}$ and satisfy $\bar{Y}_{g_{j}}=q_{Y}^{-1}\left(Y_{j}\right)$.

Now, let $\Lambda$ be a finitely generated free group of line bundles on $X$ that contains bundles $L_{1}, \ldots, L_{l}$ and sections $f_{i}: X \rightarrow L_{i}$ such that any collection $x_{1}, \ldots, x_{k} \in X$ has a common affine neighbourhood $X_{i}:=X \backslash Z\left(f_{i}\right)$. Enlarging $\Lambda$ and replacing $\Gamma$ and $\Lambda$ with suitable subgroups, we can assume that there is a canonical pullback homomorphism

$$
\varphi^{*}: \Gamma \rightarrow \Lambda, \quad E \mapsto \varphi^{*}(E) .
$$

Let $\mathcal{A}$ be the graded $\mathcal{O}_{X}$-algebra associated to the group $\Lambda$. Consider the corresponding prevariety $\widehat{X}:=\operatorname{Spec}(\mathcal{A})$ over $X$, and denote the canonical map by $q_{X}: \widehat{X} \rightarrow X$. Again $\widehat{X}$ is quasiaffine and $q_{X}$ is a geometric quotient for the action of $H_{X}:=\operatorname{Spec}(\mathbb{K}[\Gamma])$ on $X$. Note that the pullback homomorphism $\varphi^{*}: \Gamma \rightarrow \Lambda$ induces a homomorphism $\widetilde{\varphi}: H_{X} \rightarrow H_{Y}$ of algebraic tori.

Use again [5, Lemma 2.4] to choose an $H_{X}$-equivariant affine closure $\bar{X}$ of $\widehat{X}$ such that for some finite system $h_{n} \in \mathcal{B}(Y)$ of homogeneous generators of the algebra $\mathcal{O}(\bar{Y})$ the pullback sections $\varphi^{*}\left(h_{n}\right)$ extend regularly to $\bar{X}$. Additionally, ensure that the functions $f_{i} \in \mathcal{O}(\widehat{X})$ extend regularly to $\bar{X}$ and satisfy $\bar{X}_{f_{i}}=$ $q_{X}^{-1}\left(X_{i}\right)$.

So far, we have defined all the data occuring in the items (i), (ii), (iv) and (v). The only missing thing is to establish the commutative diagram of (iii). For this define a $\varphi^{*}$-graded homomorphism $\bar{\varphi}^{*}: \mathcal{O}(\bar{Y}) \rightarrow \mathcal{O}(\bar{X})$ by setting on homogeneous elements

$$
\mathcal{B}_{E}(Y) \ni g \mapsto \varphi^{*}(g) \in \mathcal{A}_{\varphi^{*}(E)}(X) .
$$

Then this homomorphism induces a morphism $\bar{\varphi}: \bar{X} \rightarrow \bar{Y}$ which is $\widetilde{\varphi}$ equivariant in the sense of item (iii). To obtain a restricted morphism $\widehat{\varphi}: \widehat{X} \rightarrow \widehat{Y}$, 
we have to verify that $\bar{\varphi}$ maps $\widehat{X}$ indeed to $\widehat{Y}$. This is true because we have

$$
\begin{aligned}
\widehat{X} & =\bigcup_{j=1}^{s} q_{X}^{-1}\left(\varphi^{-1}\left(Y \backslash Z\left(g_{j}\right)\right)\right) \\
& =\bigcup_{j=1}^{s} q_{X}^{-1}\left(X \backslash Z\left(\varphi^{*}\left(g_{j}\right)\right)\right) \\
& \subset \bigcup_{j=1}^{s} \bar{X}_{\varphi^{*}\left(g_{j}\right)}
\end{aligned}
$$

Since in degree zero, the pullback $\widehat{\varphi}^{*}$ is nothing but the usual pullback of functions on $Y$ via $\varphi: X \rightarrow Y$, also the lower part of the diagram in (iii) is commutative.

Proof of the Theorem. Choose a lifting of the morphism $\varphi: X \rightarrow Y$ and data as in the Lemma. Complete the collections $f_{1}, \ldots, f_{r} \in \mathcal{O}(\bar{X})$ and $g_{1}, \ldots, g_{s} \in$ $\mathcal{O}(\bar{Y})$ by homogeneous functions to obtain closed embeddings

$$
\begin{aligned}
& \imath_{X}: \bar{X} \rightarrow \mathbb{K}^{n_{X}}, \quad x \mapsto\left(f_{1}(x), \ldots, f_{r}(x), f_{r+1}(x), \ldots, f_{n_{X}}(x)\right) \\
& \imath_{Y}: \bar{Y} \rightarrow \mathbb{K}^{n_{Y}}, \quad y \mapsto\left(g_{1}(y), \ldots, g_{s}(y), g_{s+1}(y), \ldots, g_{n_{Y}}(y)\right)
\end{aligned}
$$

Endow the spaces $\mathbb{K}^{n_{X}}$ and $\mathbb{K}^{n_{Y}}$ with the respective diagonal actions of $H_{X}$ and $H_{Y}$ such that these embeddings become equivariant. Let $\Phi: \mathbb{K}^{n_{X}} \rightarrow \mathbb{K}^{n_{Y}}$ be a polynomial map that extends $\bar{\varphi}: \bar{X} \rightarrow \bar{Y}$ and fulfils

$$
\Phi(t \cdot z)=\widetilde{\varphi}(t) \cdot \Phi(z) \quad \text { for all }(t, z) \in H_{X} \times \mathbb{K}^{n_{X}} .
$$

Now, let $n_{X, Y}:=n_{X}+n_{Y}$, and consider the graph $\Psi: \mathbb{K}^{n_{X}} \rightarrow \mathbb{K}^{n_{X, Y}}$ of the $\operatorname{map} \Phi: \mathbb{K}^{n_{X}} \rightarrow \mathbb{K}^{n_{Y}}$. Then $\Psi \circ \imath_{X}$ embeds $\bar{X}$ equivariantly into $\mathbb{K}^{n_{X, Y}}$, where $H_{X}$ acts on the latter space via

$$
t \cdot(x, y):=(t \cdot x, \widetilde{\varphi}(t) \cdot y) .
$$

In the sequel, we regard $\bar{X}$ as a subvariety of $\mathbb{K}^{n_{X, Y}}$ and $\bar{Y}$ as a subvariety of $\mathbb{K}^{n_{Y}}$. Thus, denoting by $\operatorname{pr}_{Y}: \mathbb{K}^{n_{X, Y}} \rightarrow \mathbb{K}^{n_{Y}}$ the projection onto the second factor, we are in the following situation:

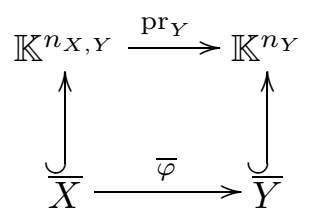

Let $W_{X} \subset \mathbb{K}^{n_{X, Y}}$ be the minimal open toric subset containing $\widehat{X}$. Analogously let $W_{Y} \subset \mathbb{K}^{n_{Y}}$ be the minimal open toric subset with $\widehat{Y} \subset W_{Y}$. Then we claim:

$$
\widehat{X}=\bar{X} \cap W_{X}, \quad \widehat{Y}=\bar{Y} \cap W_{Y}, \quad \operatorname{pr}_{Y}\left(W_{X}\right) \subset W_{Y} .
$$


For the first equation note that $\widehat{X}$ is obtained by removing the common zeroes of the first $r$ coordinates of $\mathbb{K}^{n_{X, Y}}$ from $\bar{X}$. Thus $\widehat{X}$ is the intersection of $\bar{X}$ with an open toric subset of $\mathbb{K}^{n_{X, Y}}$. Minimality of $W_{X}$ gives the desired statement. The same argument works for the second equation.

To verify the third observation, let $B_{1}, \ldots, B_{m}$ denote the orbits of the big torus $\mathbb{T}^{n_{X, Y}}$ of $\mathbb{K}^{n_{X, Y}}$ that intersect $\widehat{X}$. Then $W_{X}$ is the union of all $\mathbb{T}^{n_{X, Y}}$-orbits that have one of the $B_{l}$ in its closure. Thus, given $z=(x, y) \in W_{X}$ we have

$$
\lim _{t \rightarrow 0} \lambda(t) \cdot t_{0} \cdot z=z_{0} \in \widehat{X}
$$

with some $t_{0} \in \mathbb{T}^{n_{X, Y}}$ and some one parameter subgroup $\lambda: \mathbb{K}^{*} \rightarrow \mathbb{T}^{n_{X, Y}}$. Now, applying the projection $\operatorname{pr}_{Y}$ to the above equation yields that $y=\operatorname{pr}_{Y}(z)$ lies in $W_{Y}$, and our claim is verified.

Since $H_{X}$ acts freely on $W_{X}$ and so does $H_{Y}$ on $W_{Y}$, there exist geometric quotients $W_{X} \rightarrow Z_{X}$ and $W_{Y} \rightarrow Z_{Y}$ for these actions. Thereby the quotients spaces $Z_{X}$ and $Z_{Y}$ are smooth toric prevarieties having an affine diagonal morphism, see e.g. [5, Lemma 1.6]. Moreover, we have the following commutative diagram of toric morphisms:

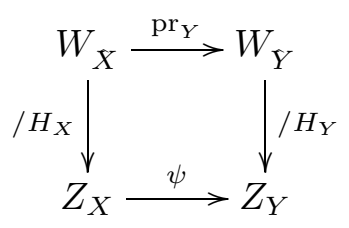

By construction, $X=\widehat{X} / H_{X}$ is embedded into $Z_{X}$ and so is $Y=\widehat{Y} / H_{Y}$ into $Z_{Y}$. By the commutative diagrams $(*)$ and statement (iii) of the Lemma, the toric morphism $\psi: Z_{X} \rightarrow Z_{Y}$ is an extension of $\varphi: X \rightarrow Y$.

It remains to show that the prevarieties $Z_{X}$ and $Z_{Y}$ are in fact $k$-divisorial. We consider exemplarily $Z_{Y}$. Since $Z_{Y}$ is smooth and has an affine diagonal morphism, it suffices to show that any $k$ points of $Z_{Y}$ admit a common affine neighbourhood in $Z_{Y}$. We do this by verifying the assumptions of [5, Corollary 4.4].

So, let $B_{1}, \ldots, B_{k} \subset Z_{Y}$ be closed orbits of the big torus of $Z_{Y}$. By minimality of $W_{Y}$, we find for each $l$ a point $y_{l} \in Y \cap B_{l}$. We construct a common affine neighbourhood of the points $y_{1}, \ldots, y_{k}$ using the method presented in [5, Proof of Theorem 5.3]:

For each $l$, choose a point $\widehat{y}_{l} \in q_{Y}^{-1}\left(y_{l}\right)$. By construction, there is a coordinate $z_{j}$ such that $\mathbb{K}_{z_{j}}^{n_{Y}}$ is a common neighbourhood of $\widehat{y}_{1}, \ldots, \widehat{y}_{k}$. Consider the closed $H_{Y}$-invariant subsets

$$
A:=\mathbb{K}_{z_{j}}^{n_{Y}} \backslash W_{\bar{Y}}, \quad \widehat{Y}_{j}:=\bar{Y} \cap \mathbb{K}_{z_{j}}^{n_{Y}}
$$

of $\mathbb{K}_{z_{j}}^{n_{Y}}$. As they are disjoint, the categorical quotient $p: \mathbb{K}^{n_{Y}} \rightarrow \mathbb{K}^{n_{Y}} / / H_{Y}$ separates these sets. In particular, no point $p\left(\widehat{y}_{l}\right)$ lies in $p(A)$. Thus we find an $H_{Y}$-invariant regular function $h$ on $\mathbb{K}_{z_{j}}^{n_{Y}}$ that vanishes along $A$ but on none of the points $\widehat{y}_{l}$. 
Removing the zero set of the function $h$ from $\mathbb{K}_{z_{j}}^{n_{Y}}$ yields a common $H_{Y^{-}}$ invariant affine open neighbourhood $U \subset W_{Y}$ of $\widehat{y}_{1}, \ldots, \widehat{y}_{k}$. Now, the image $V \subset$ $Z_{Y}$ of $U$ under the quotient map $W_{Y} \rightarrow Z_{Y}$ is the desired affine neighbourhood of the points $y_{1}, \ldots, y_{k}$.

\section{References}

[1] A. A'Campo-Neuen, J. Hausen, Toric prevarieties and subtorus actions, Geom. Dedicata 87 (2001), 35-64.

[2] M. Borelli, Divisorial varieties, Pacific J. Math. 13 (1963), 375-388.

[3] W. Fulton, Introduction to toric varieties, Annals of Mathematics Studies, 131. The William H. Roever Lectures in Geometry. Princeton University Press, Princeton, NJ, 1993.

[4] J. Hausen, A generalization of Mumford's geometric invariant theory, Doc. Math. 6 (2001), 571-592 (electronic).

[5] _ Equivariant embeddings into smooth toric varieties, Canad. J. Math. 54 (2002), $554-570$.

[6] J. Włodarczyk, Embeddings in toric varieties and prevarieties, J. Algebraic Geom. 2 (1993), 705-726.

Fachbereich Mathematik und Statistik, Universität Konstanz, 78457 Konstanz, GERMANy.

E-mail address: Florian.Berchtold@uni-konstanz.de

E-mail address: Juergen.Hausen@uni-konstanz.de 